\title{
Hipoacusia sensorioneural unilateral: Revisión de los manejos posibles en nuestra realidad nacional
}

\section{Unilateral sensorineural hearing loss: Review of possible management in our national reality}

\author{
Loreto Nicklas D. ${ }^{1,2}$, Rocío Castro $V^{3}$, Sandra Henríquez $V^{1,2}$, Constanza Mella C. $^{2}$
}

'Departamento de Especialidades Médicas, Facultad de Medicina, Universidad de la Frontera. Temuco, Chile.

¿Unidad de Otorrinolaringología, Hospital Hernán Henríquez Aravena. Temuco, Chile.

${ }^{3}$ Escuela de Medicina, Universidad de la Frontera. Temuco, Chile.

Los autores declaran no tener conflictos de interés.

Recibido el 28 de diciembre de 2020. Aceptado el 16 de marzo de 2021.

Correspondencia: Loreto Nicklas D. Dirección Casilla 3149 Portal Temuco. Temuco, Chile. Email: loreto.nicklas@ ufrontera.cl

\section{Resumen}

La hipoacusia unilateral (HUL) definida como la pérdida auditiva de cualquier grado en un solo oído tiene prevalencias en edad escolar de 3\% a 6\%. La etiología es desconocida en la mayoría de los casos, pero destacan las anormalidades anatómicas severas a diferencia de las hipoacusias bilaterales, lo que le otorga importancia al estudio de imágenes. También se recomienda realizar evaluación oftalmológica. El impacto de la HUL se observa en la localización sonora, reconocimiento del habla, desarrollo del lenguaje, desempeño social y conducta, pero por sobre todo en el rendimiento académico. En este sentido es relevante mencionar que además de existir mayor probabilidad de repitencia de curso, es necesario mayor apoyo educacional individualizado para evitar dicha repitencia o lograr mismo éxito académico que un normoyente. En relación al diagnóstico aún faltan normas o protocolos, siendo éste un desafío en las zonas donde no hay screening universal. Se discute la importancia de las encuestas para realizar evaluaciones y seguimiento en estos casos. Finalmente se abordan las estrategias de intervención disponibles, además de un plan individualizado considerando al niño, su familia y sus expectativas, particularmente se discuten las características o factores a tomar en cuenta en el momento de la adaptación, para así lograr un tratamiento tendiente al éxito.

Palabras clave: Hipoacusia unilateral, ambliaudia, sordera unilateral, sistema de micrófono remoto, CROS.

\begin{abstract}
Unilateral hearing loss (UHL) defined as hearing loss of any degree in only one ear has a school-age prevalence of 3\% to 6\%. The etiology is unknown in most cases, but severe anatomical abnormalities stand out as opposed to bilateral hearing loss, which gives importance to the study of images. Ophthalmological evaluation is also recommended. The impact of UHL is observed in sound localization, speech recognition, language development, social performance and behavior, but above all in academic performance. In this sense, it is relevant to mention that in addition to having a higher probability of repeating the course, it is necessary to have more individualized educational support to avoid this repetition or achieve the same academic success as a normal listener. Regarding the diagnosis, there are still missing norms or protocols, this being a challenge in areas where there is no universal screening. The importance of surveys to carry out evaluations and follow-up in these cases is discussed. Finally, the available intervention strategies are discussed, in addition to an individualized plan considering the child, his family and his expectations, particularly, the factors to be taken into account at the time of adaptation, in order to achieve a success treatment.
\end{abstract}

Keywords: Unilateral hearing loss, amblyaudia, single sided deafness, remote microphone system, CROS. 


\section{Introducción}

En la actualidad existen múltiples controversias al hablar de hipoacusia unilateral, especialmente en el rango de frecuencias involucradas de lo que consideramos hipoacusia, en el impacto o consecuencias en el desempeño general y calidad de vida, en los instrumentos de medición objetiva y funcional utilizados, y en el tratamiento más adecuado según cada caso. Por otra parte, se trata de un grupo de pacientes diversos según el tipo o grado de la hipoacusia, la edad del paciente, su nivel sociocultural y cognitivo e incluso según el lado afectado.

La hipoacusia unilateral (HUL) puede ser definida tan ampliamente como la pérdida auditiva de cualquier tipo en un oído ${ }^{1}$, incluyendo los casos en que exista audición no funcional, denominada sordera o cofosis unilateral. Esta definición incluye un universo de entidades que no son comparables ni uniformes entre ellas. Por un lado, tenemos un grupo de personas, por lo general ya adultas, con HUL poslingual, en donde las conexiones neurales fueron establecidas en su tiempo y por lo tanto existe un equilibrio en la configuración central de la audición, y donde el problema prevalente pareciera ser la falla en la localización sonora y la percepción del habla en ruido ${ }^{2,3}$. Por otra parte, están las hipoacusias unilaterales leves, entre 20 y $30 \mathrm{~dB}$ o las que comprometen solo las frecuencias altas sobre los $4000 \mathrm{~Hz}$ manteniendo un promedio tonal puro (PTP) normal, que son un desafío no solo en su diagnóstico oportuno, sino que, también en el manejo adecuado a edades tempranas, desconociéndose el real impacto que tienen en las personas. Otro grupo está compuesto por las hipoacusias de conducción unilateral que tienen cóclea funcionante en las que, si se logra un estímulo adecuado en el tiempo adecuado, se pudiera conseguir una audición binaural cercana a la normal ${ }^{2}$, aunque aún es controversial la aparición de ambliaudia en este grupo y después de cuanto tiempo ${ }^{4}$. Aquí también se incluyen las malformaciones del oído externo que son fácil y precozmente identificables, o todo tipo de otitis media persistente o recurrente y en las cuales los esfuerzos terapéuticos no suponen tanta duda, pero es imperante que sean realizados a tiempo. Las malformaciones unilaterales de oído externo merecen un capítulo aparte de análisis en relación a la indicación y también aceptación del tratamiento por parte del paciente o sus padres o cuidadores. Finalmente tenemos el grupo de hipoacusias sensorioneurales unilaterales que no se detectaron al nacer, aparecieron en el transcurso del tiempo o que fueron desestimadas por existir "otro oído" que supliera ambas funciones. Es en este grupo en el que queremos centrar la atención de este artículo.

Si observamos las publicaciones más precoces al respecto que decían que "la preocupación de los audiólogos y otorrinolaringólogos sobre la HUL no es otra que identificar su etiología y asegurarles a los padres que no habrá discapacidad" ", entenderemos que no se comprendía el real impacto de poseer un oído único, idea que se mantuvo, lamentablemente, hasta hace pocos años, considerándose la HUL una alteración mínima sin impacto en la vida. Tanto que ninguna política pública en salud de nuestro país considera su detección precoz o tratamiento a la fecha. Posteriormente avanzando a la década de los 90 y con la mejoría del diagnóstico precoz y universal, además de la aparición de nuevos trabajos publicados, es que aparece la idea de que las HUL deben resolverse o adaptarse precozmente ${ }^{5}$.

Previo a la utilización de algún tipo de tamizaje auditivo (1986), solo el $23 \%$ de las HUL se identificaban antes de los 5 años. Con el screening neonatal bajó la edad promedio de diagnóstico entre 2,6 y 0,3 años de edad ${ }^{6,7}$. Algunas publicaciones sugieren la implementación de un screening en edad prescolar, puesto que no todos los casos son detectables al nacer ${ }^{6,8}$.

Es difícil dar cifras de prevalencia dado lo heterogéneo de las publicaciones que no se ciñen a una única norma de reporte, tanto por la definición de HUL y la obtención de umbrales auditivos, como por los grupos donde se estima ${ }^{9}$. La prevalencia ajustada estimada en edad escolar es de $3 \%$ al $6 \%{ }^{8,10,11}$, mientras que se estima una incidencia aproximada de 0,6 a 0,7/1.000 en recién nacidos vivos según lo reportado por "Center for Disease Control and Prevention" en Estados Unidos ${ }^{12}$. Entre un $22 \%$ a $36 \%$ de las hipoacusias diagnosticadas cada año son $\mathrm{HUL}^{13}$, y otro dato importante a considerar, es que $11 \%-40 \%$ de las HUL al 
nacer progresarán en el mismo oído ${ }^{7,13-15}$. Se reportan prevalencias tan altas como un 14\% en el grupo de 12 a 19 años $^{16}$, y un $7,5 \%$ a $20 \%$ desarrollarán hipoacusia bilateral en el tiem$\mathrm{po}^{12-15}$. Dependiendo del umbral que se utilice para denominar la hipoacusia, la prevalencia de HUL cambia, reportándose que entre adolescentes habría un $14 \%$ de casos si se considera un PTP de $15 \mathrm{~dB}$ para el diagnóstico, pero solo de un $2,7 \%$ si se considera un PTP de $25 \mathrm{~dB}^{16}$.

\section{Etiología}

Dentro de las causas de HUL, y al igual que lo que ocurre con las bilaterales, existe un grupo cuya etiología es desconocida, sin embargo, a través del tiempo este grupo disminuye por la aparición de nuevas herramientas diagnósticas. Se observan causas traumáticas, posteriores a infecciones virales congénitas como parotiditis, sarampión, rubéola o citomegalovirus (CMV), meningitis, tumores, ototoxicidad, además de todas las causas de hipoacusias conductivas que no se tratan adecuadamente y persisten en el tiempo ${ }^{17}$. Las causas genéticas predominan en las hipoacusias bilaterales, pero también son responsables de algunos casos unilaterales, sobre todo cuando existe malformación a nivel del hueso temporal. Un 30\%-41\% de los casos presentan anormalidades estructurales del hueso temporal en tomografía computarizada (TC), sin embargo, a menos de la mitad de los pacientes portadores del HUL severa se les solicitan imágenes en su estudio ${ }^{18,19}$.

Dentro de las anomalías de hueso temporal se describen: acueducto vestibular dilatado (EVA) en un 14\%-23\% de las HUL (que frecuentemente se presenta en forma bilateral y podría explicar el compromiso del oído contralateral en el tiempo), aplasia o hipoplasia del nervio coclear en aproximadamente un $50 \%$ de las HUL profundas versus un 5\% de las hipoacusias bilaterales, existiendo reportes que aseguran prevalencias más altas ${ }^{17,20,21}$, malformaciones cocleovestibulares diversas, siendo la más frecuentemente encontrada la malformación de Mondini, describiendo que a más profunda la hipoacusia más severa es la anormalidad $^{15,22}$. Igualmente, es importante considerar que la HUL coexiste en un $67 \%$ con lesiones oftamológicas concomitantes que incluyen displasias o aplasias de nervio óptico ${ }^{21}$. Con lo anterior cabe cuestionarse si en el estudio de estos pacientes bastará con un TC para evaluar estructuras óseas o sería necesario además solicitar una resonancia magnética $(\mathrm{RM})^{14,23}$, la que seguramente no será fácil conseguir por la edad de los pacientes y la necesidad de anestesia o su disponibilidad en los distintos centros.

\section{Impacto en el desempeño}

Considerando que las ventajas de la binauralidad incluyen la localización sonora, reconocimiento de la palabra/habla, sumación binaural, audición en ruido; de esta forma, las personas portadoras de una HUL tendrán marcados problemas en estos aspectos ${ }^{24,25}$. Se ha evidenciado varias consecuencias como alteración del lenguaje en preescolares ${ }^{26,27}$, bajo desarrollo socioemocional y autoestima, alteración en los logros académicos donde se describe que entre un cuarto y la mitad de los niños con HUL tienen dificultades como alteración en la retención, necesidad de clases adicionales de reforzamiento y reportes de profesores que denuncian en estos niños mayor tendencia a la distracción, desatención, aislamiento e incluso agresividad ${ }^{28,29}$. Además, existen retrasos en las competencias del habla y lenguaje en un $25 \%$ a $40 \%$ que se supera en el tiempo ${ }^{30}$, y alteraciones en ciertas funciones cognitivas como menor coeficiente intelectual (CI) verbal ${ }^{30}$ o disminución en el llamado "multitask" o la habilidad de ejecutar varias tareas al mismo tiempo ${ }^{31}$. Por otra parte, se ha identificado que, al fallar la localización, los niños deben utilizar la visión para identificar la fuente sonora ${ }^{31,32}$.

Lo anterior se suma a que la mayoría de las clases escolares en nuestro país suponen para sus alumnos tareas simultáneas como escuchar, pensar respuestas, responder, tomar notas, ver afiches o imágenes, lo que agregado a ambientes auditivamente complejos como son las salas de clases, traducen para aquellos que tienen HUL un esfuerzo adicional y, por tanto, fatiga ${ }^{31,32}$.

Desde hace muchos años atrás se estudian sus consecuencias a nivel educacional, reportándose que existe 10 veces más repitencia que los pares normoyentes, con $35 \%$ a $20 \%$ de los escolares con HUL repitiendo al menos 
un curso versus $3,5 \%$ a $2 \%$ de los normoyentes ${ }^{29,33,34}$. Asimismo, los niños con HUL tienen 4,4 veces más necesidad de reforzamiento o apoyo educacional individualizado que sus pares normales para evitar dicha repitencia, lo que se mantiene en el tiempo ${ }^{29,30,35}$ y se estima que un 59\% de niños con HUL tienen algún problema académico o de conducta ${ }^{5}$. Al aplicar encuestas de calidad de vida enfocadas en ambientes auditivos y que evalúan aspectos como el desempeño ante familia y amigos, actividades recreacionales, escuela y sentimientos, los reportes señalan que un hipoacúsico unilateral tiene alteraciones en su calidad de vida más similares a un hipoacúsico bilateral que a un normoyente $28,36,37$.

Ante la pregunta de si existen diferencias según el oído afectado, se ha observado compromiso más notorio en aspectos como desarrollo académico, repitencia de curso, retraso del habla y del lenguaje, pero por sobre todo reorganización cerebral cuando existe compromiso del oído derecho (OD) $)^{25,38,39}$. Sin embargo, lo más importante es evitar la ambliaudia y sus consecuencias ${ }^{40}$, la cual es provocada por la deprivación auditiva unilateral que puede distorsionar los mapas tonotópicos, alterar la integración binaural, reorganizar las redes neuronales, y cambiar la transmisión sináptica en la corteza auditiva primaria o región subcortical. Lamentablemente la lateralización de las vías auditivas es difícil de revertir cuando ya está instaurada ${ }^{40,41}$.

\section{Opciones de manejo}

En la actualidad se describen alternativas como la simple observación y seguimiento del paciente, intervención o apoyo fonoaudiológico, instaurar clases de reforzamiento y otras medidas escolares como el asiento preferencial cercano a la fuente sonora o voz del profesor, pero también están disponibles varias opciones de amplificación ${ }^{15,38}$.

Es importante sugerir la modificación de la distribución de las clásicas salas de clases que ordenan los puestos de trabajo en filas y columnas, lo que no solo puede ser un obstáculo a la propagación de la onda sonora para las ubicaciones más posteriores, sino que se suma la dificultad de visualizar al profesor o al recurso audiovisual expuesto. Las ubicaciones cercanas a las paredes se someten a más reverberancia, lo que es mayor si sumamos superficies, cielos y pisos lisos sin amortiguación acústica, determinando ambientes auditivamente complejos. También es aconsejable menor número de estudiantes por sala, medida no fácil de adoptar por el costo que implica ${ }^{32,42}$ (Figura 1).

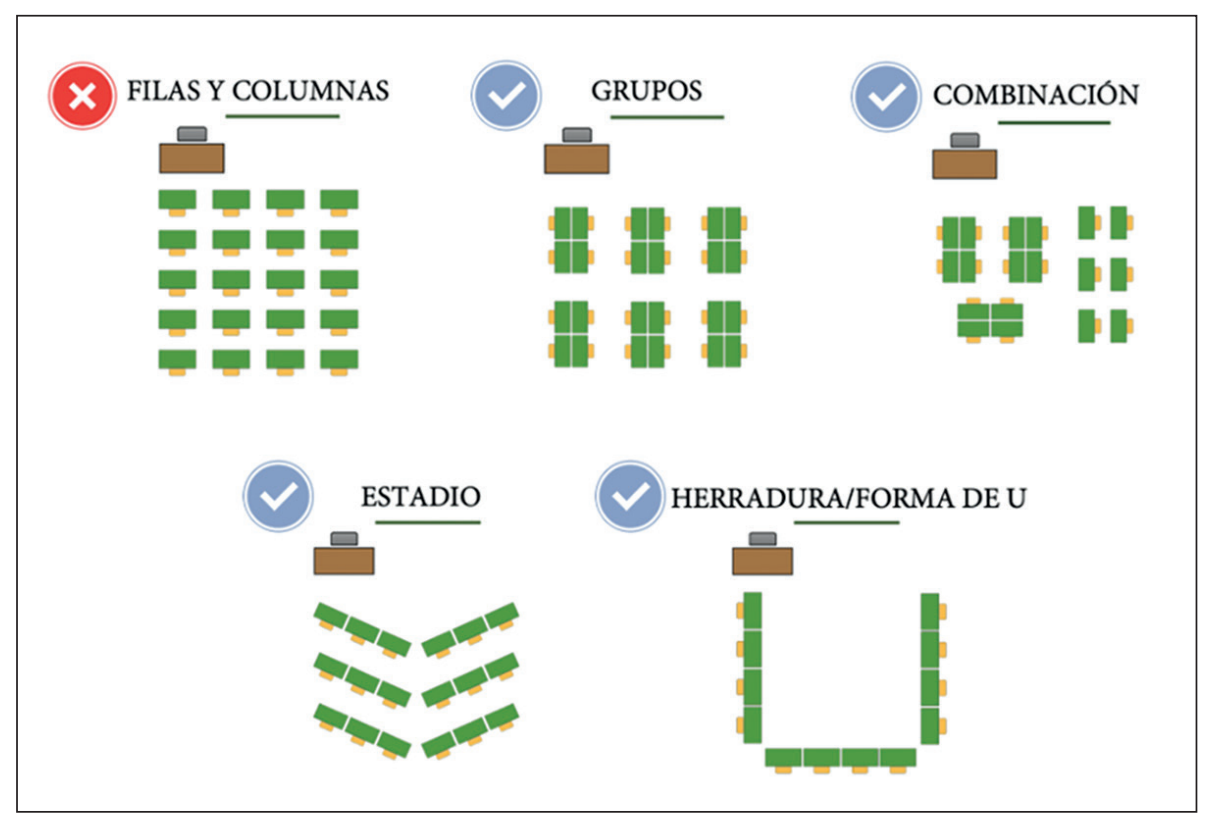

Figura 1. Formatos de distribución de salas o aulas de clases, en donde configuraciones distintas a la tradicional de filas y columnas, pueden mejorar las condiciones auditivas. 
El apoyo, seguimiento, terapia fonoaudiológica, y en muchos casos, el complemento multidisciplinario con educación diferenciada especialista, terapia ocupacional y psicopedagógica, es parte de la base del tratamiento y es el pilar fundamental que regula todos los aspectos que se exponen en esta revisión. La participación activa del colegio y capacitación del profesorado, también son de gran importancia ${ }^{30,35}$.

Dentro de las tecnologías disponibles para HUL tenemos: audífonos tradicionales, sistemas de frecuencia modulada (FM), o mejor denominados como sistemas de micrófono remoto (SMR), sistemas de "derivación contralateral de la señal”, llamados en inglés contralateral routing of signal (CROS), dispositivos o implantes de conducción Ósea y el implante coclear (IC) $)^{15,38,42,43}$.

Todos los dispositivos mencionados anteriormente y el apoyo fonoaudiológico aún no están contemplados ni financiados por el programa GES de nuestro país para la HUL. Para el grupo de escolares entre los 5 y 18 años, siempre y cuando el establecimiento educacional haya inscrito al alumno, existe la posibilidad de algunas ayudas auditivas por la Junta Nacional de Auxilio Escolar y Becas (JUNAEB), solo si el otorrino de dicha institución lo considera pertinente. Cabe notar que a esas edades la implementación es tardía en la mayoría de los casos ${ }^{44}$.

Llama la atención que en la práctica clínica se reporta una limitada indicación de audífonos al momento del diagnóstico de las HUL (aproximadamente un 25\%) a diferencia de los casos diagnosticados como hipoacusia leve o sólo de alta frecuencia bilaterales ${ }^{45}$, lo que puede explicarse por la idea de que las madres de niños con hipoacusia leve o unilateral reportan que les es difícil aceptar la necesidad de amplificación, porque sus hijos responden a muchos sonidos con o sin ella ${ }^{46}$.

En relación con el uso de audífonos convencionales, la evidencia indica que se deben implementar en el oído hipoacúsico cada vez que exista audición residual utilizable ${ }^{47}$ y lo más precozmente posible. Se describe que en niños bastaría con la sola detección del sonido para determinar el ambiente sonoro que los rodea $^{15,38}$. En caso de que dicha implementación no sea precoz, y por ende se pierdan las conexiones centrales y la discriminación, no hay estudios que muestren beneficios del audífono en HUL que superen la molestia que ocasiona a los padres el tratar que los niños no se los saquen ${ }^{43}$. Sin embargo, se debe considerar que existe mejor desempeño social y académico, además de facilidad auditiva y mejor localización sonora al implementar el oído hipoacúsico. De todas maneras, se debe considerar el porcentaje de hipoacusia profunda reportado en HUL por malformaciones severas que dificultaría la implementación y hace necesario buscar otras opciones. Lamentablemente en este ámbito falta información para mejorar la correcta prescripción ${ }^{47}$. Está pendiente determinar una fórmula de adaptación del audífono, dado que pareciera que la convencional para pérdidas bilaterales no sería la correcta ${ }^{38,42}$. Se deben considerar los factores de riesgo para un mal desempeño académico en una persona portadora de HUL que se reporta en un $41 \%^{48}$, en los cuales se debe recalcar aún más la implementación de ayudas auditivas y necesidad de apoyo escolar o reforzamiento. Dicho desempeño es peor dependiendo de factores como el nivel socioeconómico, instrucción materna y factores asociados al paciente como oído afectado, grado de hipoacusia y CI nativo verbal bajo (refiriéndose como CI nativo a la puntuación verbal lograda por factores no dependientes de los efectos de la hipoacusia, que pueden alterar las habilidades verbales) ${ }^{30,35}$.

Para los casos de hipoacusia permanente moderada o severa con buena discriminación y detectada precozmente, no hay duda en la indicación de un audífono como primera línea de tratamiento, pero los casos mínimos o leves son un desafío que debe solucionarse en forma multidisciplinaria y en conjunto con los padres, en donde la adaptación de un posible audífono debe ser analizada caso a caso. Si la hipoacusia es profunda, el audífono convencional deja de ser una solución y se deben analizara otras opciones como las que expondremos a continuación ${ }^{15}$.

Otras ayudas auditivas para HUL incluyen los sistemas de micrófono o amplificación remotos, generalmente conocidos como equipos FM. Este concepto tan amplio involucra desde un micrófono en la fuente sonora y un parlante (o receptor) que puede ubicarse en 
un audífono, en un auricular sin amplificación en el oído sano (por lo tanto, no un audífono propiamente tal, el cual, para evitar el efecto de oclusión, debe tener formato abierto), o en headphones o auriculares supraurales convencionales, hasta sistemas de parlantes individuales en el escritorio del estudiante o sistemas más inclusivos e ideales como serían parlantes alrededor de la sala de clases ${ }^{15,42}$. Esta última modalidad además estaría indicada en niños con déficit atencional, y en nuestro país ha sido poco explorada y poco utilizada. Con este sistema se disminuirían los efectos negativos del ruido ambiental, la distancia y la reverberación de las salas de clases, sin embargo, existen pocos estudios que demuestren beneficios y con baja casuística y no enfocados en HUL ${ }^{49,50}$. Este sistema requiere capacitación activa del profesorado en su uso, de lo que puede encargase el fonoaudiólogo o educador especialista responsable de la rehabilitación auditiva del niño. Se debe tomar en cuenta la edad del niño y la calidad auditiva del oído hipoacúsico implementado, puesto que una alternativa sería colocar un receptor (no audífono) no oclusivo en el oído sano.

Con la evidencia publicada hasta ahora puede considerarse el mejor sistema para mejorar la relación señal/ruido ${ }^{49,51}$. Su diseño es especial para el aula con clases expositivas. En el caso de los lactantes o prescolares, se debe analizar caso a caso, pero podrían recomendarse en ciertas instancias de la vida diaria como traslados en auto, en entretenimiento o trabajo frente a pantallas o al ingresar a la educación prescolar siempre y cuando exista colaboración y capacitación del educador. El SMR puede también ser una alternativa en todos los grados de hipoacusia: pudiendo complementar a la adaptación con audífono durante las clases expositivas o en trabajo en sala de clases y en los casos en que se decida no implementar un audífono por ser hipoacusia leve, o en la hipoacusia profunda, utilizando el oído sano ${ }^{15,42,51}$.

Otra alternativa auditiva es el CROS, que ha mostrado beneficios en ambientes no ruidosos, es útil cuando no hay acceso a micrófono remoto o en actividades extraaula, pero solo recomendados en adultos o cuando los niños son capaces de manejar su ambiente sonoro o de comunicación para disminuir ruido hacia el oído sano y así mejorar la señal sonora $^{15,38,52}$. Los resultados publicados son variables y dependen tremendamente del ambiente auditivo ${ }^{51,53}$. Es importante considerar que aún falta determinar si el uso de un CROS pudiera alterar la compensación en la localización sonora lograda por el único oído, no solo no lográndola (dado que al existir una cóclea estimulada es imposible mejorarla), sino que incluso empeorándola, dado que podría interferir con la información sonora adquirida por dicho oído, a lo que se suma un posible efecto de oclusión, cosa que podría evitarse con un dispositivo de derivación contralateral implantable o el uso de formato abierto ${ }^{41}$.

En relación a los dispositivos antes mencionados, CROS y SMR, existe poca evidencia para poder recomendar uno u otro en HUL, y ante la pregunta de cuál tipo de amplificación tiene más ventajas, solo existen dos estudios con 6 sujetos cada uno, que muestran tendencia a recomendar más los sistemas de micrófono remoto dado que producen mejor reconocimiento del habla o captación de la señal en variadas condiciones auditivas incluyendo el ruido ambiental ${ }^{49,50}$.

La adaptación auditiva osteointegrada, generalmente indicada para atresia de conducto auditivo externo, tiene utilidad en HUL sensorioneural profunda por la derivación contralateral de la información auditiva por vía ósea. Los estudios disponibles muestran resultados diversos, no comparables entre ellos, claramente insuficientes para poder emitir recomendaciones con precisión y aún son escasos en niños ${ }^{51}$. Han sido indicados en adultos describiendo mejorías en: la calidad de vida, desempeño en ruido, índices de reconocimiento del habla y en la localización, esto último solo en los casos de hipoacusia de conducción y no así en hipoacusia sensorioneural como puede inferirse al existir solo una cóclea estimulada ${ }^{52,54}$. Últimamente se reportan mejoría de la percepción del habla y de la memoria en niños con este tipo de dispositivos en HUL sensorioneural, pero obviamente sin mejoría de la localización sonora y con mal desempeño en ambientes ruidosos ${ }^{38,52,54}$. Los estudios que muestran seguimiento, describen una relativa baja en la adherencia en el tiempo, aunque los tiempos de seguimiento aún son insuficientes para poder emitir conclusiones ${ }^{51}$. Esto pudie- 
ra explicarse, porque en adultos solo $1 / 3$ de las HUL sensorioneurales reportan obtener beneficios a largo plazo ${ }^{38}$. Por otra parte, se sugiere que en niños pequeños menores de 5 años (FDA no recomienda implantes antes de esta edad) o en adultos previos al implante, primero utilicen un vibrador óseo, justamente para demostrar beneficios y adherencia antes de pasar a la etapa de implante ${ }^{38,51,52}$. Las revisiones a la fecha concluyen que la decisión terapéutica varía caso a caso, pero que un implante osteointegrado de vibrador óseo traería los beneficios antes mencionados ${ }^{42,51}$.

Finalmente, en relación a los IC la evidencia es escasa, pero promisoria, aun con casuísticas bajas, la mayoría en adultos, nivel de evidencia bajos, las variables medidas y expresadas son muy diversas o heterogéneas ${ }^{53}$ y en el caso de los niños es difícil objetivar los resultados de los instrumentos de medición auditiva ${ }^{38}$. Existen revisiones sistemáticas y metaanálisis ${ }^{53,55,57}$ que concluyen que los beneficios se observan solo en las hipoacusias agudas poslinguales, se recalca la importancia en la mejoría del tinnitus ${ }^{63}$ y finalmente se amplía el uso de IC en HUL, pero los niveles de evidencia son bajos hasta la fecha. Existen mejores resultados mientras más precoz el implante ${ }^{55}$ y se debe tener en cuenta que es necesario desarrollar criterios selectivos de implante en niños con HUL, dada la alta prevalencia de aplasia o hipoplasia del nervio coclear ${ }^{15,57}$. Los últimos reportes en niños, enfatizan en indicarlos tempranamente cuando existen periodos cortos de deprivación auditiva, antes de que se produzca la reorganización cerebral, puesto que la ambliaudia producida no permitirá conseguir todas las ventajas de la binauralidad, especialmente la localización sonora ${ }^{2,25,64}$. Muestran buena adherencia en el tiempo ${ }^{15,64}$, ventajas auditivas y subjetivas, mejor calidad de vida, pero siempre advierten que aún falta más información al respecto y estudios con más casuística ${ }^{41,47,58-61}$, sin embargo, hay otros reportes que indican que el comportamiento cortical y plasticidad neuronal pudieran ser tolerantes y diferentes en las en HUL neurosensorial congénita en relación a la hipoacusia bilateral y, por tanto, el tiempo disponible para implante pudiera ser diferente o requerir de otras herramientas para la habilitación auditiva ${ }^{59,62}$. Aún no está establecida la ventana definitiva de mejor edad para implante en estos pacientes, aunque se supone que es corta, está pendiente definir el tiempo a considerarse precoz o tardío, pero deben utilizarse los mismos parámetros temporales que para los implantes de los casos bilaterales ${ }^{24}$. Finalmente, surgen nuevos métodos e ideas para evaluar la reorganización cerebral por HUL prolongada, tanto de pruebas audiológicas de localización y discriminación, potenciales evocados corticales e imágenes funcionales complejas que pueden ayudar a emitir un pronóstico de rehabilitación auditiva, antes de intentar implantar definitivamente una posible ambliaudia $^{41}$.

En relación con la evaluación de resultados auditivos funcionales de las distintas intervenciones en HUL y la recolección de evidencia de poder estadístico para poder emitir recomendaciones $^{53}$, es importante recalcar la comunicación de estos resultados con encuestas debidamente validadas en nuestro entorno e idioma, aplicables a pacientes de todas las edades, sus padres, cuidadores y profesores. También es importante obtener medidas auditivas objetivas y estandarizadas como pruebas de puntajes de reconocimiento de sílabas, palabras y/o frases validadas en nuestro medio ${ }^{51}$, de lo cual hay poco material local.

No debemos olvidar en el manejo proteger el oído contralateral del trauma acústico, manejar patologías a nivel de oído medio que comprometen, aunque sea parcialmente la audición y monitorear la posible progresión y bilateralización de la hipoacusia ${ }^{13,14}$. Por lo anterior se debe ajustar la ayuda auditiva recomendada y se debe otorgar información a padres o cuidadores de la importancia de la intervención temprana y seguimiento del niño para que así puedan tomar decisiones informadas.

\section{Discusión}

Es necesario considerar el impacto de esta patología y por tanto la implementación de políticas públicas en nuestro medio para el diagnóstico, apoyo y manejo de los portadores de HUL, incluyéndola no solo en el tamizaje neonatal, sino que además en un posible cribado preescolar ${ }^{6,8}$.

Debemos recalcar que, en HUL no se debe 
olvidar una probable progresión de la hipoacusia y posible compromiso contralateral en el tiempo, en especial en los casos en que se diagnostique un EVA o cuando la hipoacusia fue causada por $\mathrm{CMV}^{13}$. El estudio idealmente debería considerar RM de fosa posterior y TC de oídos. No olvidar solicitar evaluación oftalmológica, sobre todo cuando hay lesiones de nervio coclear demostradas en imágenes (Tabla 1).

Es importante además monitorear posible compromiso del oído medio del oído sano y dar tratamiento en forma oportuna, así como la instrucción de protección ante trauma acústico. Otros esfuerzos se deben enfocar en validar o crear, según nuestra realidad nacional, distintos instrumentos de medición auditiva funcional como encuestas o valoraciones de reconocimiento de sílabas, palabras o habla, tanto para fines diagnósticos como de seguimiento terapéutico de estos casos.

Sería apropiado plantearse la modificación de la distribución espacial de los puestos de los estudiantes en la sala de clases a otros que fomenten la visión del expositor y que acústicamente sean más amigables (Figura 1). Ideal sería dictar normas nacionales para que todas las aulas de clases tengan estas características, que además incluyan paredes y superficies con amortiguación acústica, incluso con la instalación de amplificación sonora en las salas de clases de ser necesario, logrando así un manejo inclusivo de los afectados ${ }^{38,42}$.

Es fundamental intentar la implementación lo más precoz posible y apoyar con un equipo multidisciplinario para así intervenir el entorno académico sobre todo en los casos de compromiso del oído derecho, bajos nivel socioeconómico o instrucción materna y cuando existe compromiso de las pruebas de CI verbal. En estos casos, considerar y sugerir la posible necesidad de educación personalizada o clases de reforzamiento.

Las indicaciones de las distintas ayudas auditivas aún están en constante evolución, y es necesario obtener más información al respecto, a medida que se publican reportes con más y mejores casuísticas e idealmente con información local.

En HUL sensorioneural se debe otorgar un plan individualizado considerando al niño, su familia y sus expectativas. Con los datos revisados, se propone un esquema de manejo, siendo la implementación con la mayor premura posible de un audífono o un implante coclear, según sea el grado de la hipoacusia, las mejores opciones de manejo en caso de tener un diagnóstico precoz y consenso con los padres o paciente, siempre con la base del apoyo fonoaudiológico y multidisciplinario. En los casos tardíos, considerar sistemas que refuercen al oído contralateral sin ocluirlo y faciliten la adquisición de información auditiva como los SMR o CROS (aéreo o implantable) según sean las condiciones detalladas en esta revisión y los hallazgos de los próximos estudios (Figura 2).

Debemos tener en cuenta que, en contraste con HUL aguda en adulto, un niño con HUL congénita no nota el déficit y en la adolescencia tampoco admiten el impacto, porque no quieren ser diferentes, es así como los padres, finalmente, consultan por los problemas conductuales, de interacción social o de personalidad incluso por la preocupación de un tumor más que por la hipoacusia en sí, por lo que se

Tabla 1. Recomendaciones para el estudio de $\mathrm{HUL}^{15}$

\section{Estudio inicial}

1. Estudio de imágenes (TC y RM)

2. Evaluación oftalmológica

3. Evaluación genética (sobre todo en sospecha de algún síndrome o desorden de espectro de neuropatía auditiva)

4. Examen de detección de infección por CMV

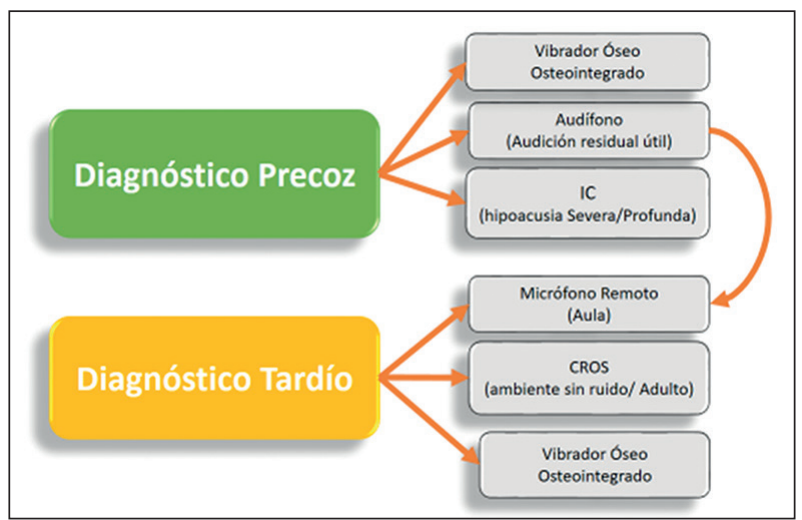

Figura 2. Esquema de manejo sugerido en base al momento del diagnóstico y según los recursos disponibles. 
dificulta la adherencia al manejo indicado. En el tratamiento de HUL cobra importancia el ámbito estético, lo que en edades tempranas puede manejarse con colores o diseños atractivos para que sean equipos estéticamente amigables, pero para otras edades los avances tecnológicos deben apuntar a que la ayuda auditiva sea lo menos visible posible.

Considerar en los adultos con HUL que las etiologías son amplia y fácilmente conocidas, no presentan impacto en lenguaje, el tratamiento se otorgará según el tiempo y grado de hipoacusia, el área de desempeño o el tinnitus y en ellos, al manejar bien su ambiente auditivo, CROS es una buena opción. Queda en el futuro evaluar los beneficios de la osteointegración en este ámbito y en los casos de HUL sensorioneural precoces y profundos el IC. Un buen predictor de la adherencia al uso de ayudas auditivas son las actividades sociales y laborales, la comodidad y confort que brinda la tecnología y conectividad digital actual ${ }^{38}$. Aun es mucha la información faltante para poder tomar mejores decisiones.

\section{Bibliografía}

1. Bess F, Tharpe A. An introduction to unilateral sensorineural hearing loss in children. Ear Hear. 1986;7(1):3-13.

2. Gordon K, Henkin Y, Kral A. Asymmetric Hearing During Development: The Aural Preference Syndrome and Treatment Options. Pediatrics. 2015;136(1):141-53. doi: 10.1542/peds.2014-3520.

3. Kim G, Mi Ju H, Hee Lee S, Kim H, Kwon J, Seo Y. Efficacy of Bone-Anchored Hearing Aids in Single-Sided Deafness: A Systematic Review. Otol Neurotol. 2017;38(4):473-483. doi: 10.1097/ MAO.0000000000001359.

4. Northern J, Downs M. Hearing In Children. 2th ed. Baltimore: Williams \& Wilkins Co; 1978.

5. Brookhouser P, Worthington D, Kelly W. Unilateral hearing loss in children. Laryngoscope. 1991;101(12):1264-1272.

6. Ghogomu N, Umansky A, Lieu J. Epidemiology of unilateral sensorineural hearing loss with universal newborn hearing screening. Laryngoscope. 2014;124(1):295-300.

7. Fitzpatrick E, Whittingham J, Durieux-Smith A. Mild bilateral and unilateral hearing loss in childhood: a 20 -year view of hearing characteristics, and audiologic practices before and after newborn hearing screening. Ear Hear. 2014;35(1):10-18.
8. Ross D, Holstrum W, Gaffney M, Green D, Oyler R, Gravel J. Hearing screening and diagnostic evaluation of children with unilateral and mild bilateral hearing loss. Trends in Amplification. 2008;12(1): 27-34.

9. Lieu J. Variations in the Prevalence of Hearing Loss in Children: Truth or Artifact? JAMA Otolaryngol Head Neck Surg. 2017;143(9):935-936. doi: 10.1001/ jamaoto.2017.1172.

10. Zeitler D, Sladen D, DeJong M, Torres J, Dorman M, Carlson M. Cochlear implantation for single-sided deafness in children and adolescents. Int J Pediatr Otorhinolaryngol. 2019;118:128-133.

11. Ross D, Visser S, Holstrum W, Qin T, Kenneson A. Highly variable population-based prevalence rates of unilateral hearing loss after the application of common case definitions. Ear Hear. 2010;31:126-133.

12. Disease Control Early Hearing Detection and Prevention [CDC] Database. National Workshop on Mild and Unilateral Hearing Loss (2005). Centers for Disponible en: https://www.cdc.gov/ncbddd/ hearingloss/conference.html. Consultado el 18 de febrero de 2021.

13. Lieu J. Permanent Unilateral Hearing Loss (UHL) and Childhood Development. Curr Otorhinolaryngol Rep. 2018;6(1):74-81.

14. Paul A, Marlin S, Parodi M, et al. Unilateral Sensorineural Hearing Loss: Medical Context and Etiology. Audiol Neurootol. 2017;22(2):83-88.

15. Bagatto M, DesGeorges J, King A, et al. Consensus practice parameter: audiological assessment and management of unilateral hearing loss in children. Int J Audiol. 2019;58(12):805-815.

16. Shargorodsky J, Curhan S, Curhan G, Eavey $\mathrm{R}$. Change in prevalence of hearing loss in US adolescents. JAMA. 2010;304(7):772-778.

17. Laury A, Casey S, McKay S, Germiller J. Etiology of unilateral neural hearing loss in children. Int J Pediatr Otorhinolaryngol. 2009;73(3):417-427.

18. Dodson K, Georgolios A, Barr N, et al. Etiology of unilateral hearing loss in a national hereditary deafness repository. Am J Otolaryngol. 2012;33(5):590-594.

19. Friedman A, Guillory R, Ramakrishnaiah R, et al. Risk analysis of unilateral severe-to-profound sensorineural hearing loss in children. Int J Pediatr Otorhinolaryngol. 2013;77(7):1128-1131.

20. Nakano A, Arimoto Y, Matsunaga T. Cochlear nerve deficiency and associated clinical features in patients with bilateral and unilateral hearing loss. Otol Neurotol. 2013;34(3):554-558.

21. Clemmens C, Guidi J, Caroff A, et al. Unilateral cochlear nerve deficiency in children. Otolaryngol Head Neck Surg. 2013;149(2):318-325.

22. Fitzpatrick E, Al-Essa R, Whittingham J, Fitzpatrick J. Characteristics of children with unilateral hearing loss. Int J Audiol. 2017;56(11):819-828.

23. Waissbluth S, Bravo S, Gutiérrez C. Estenosis 
congénita aislada del conducto auditivo interno como causa de hipoacusia neurosensorial unilateral y paresia del nervio facial. Rev Otorrinolaringol Cir Cabeza Cuello. 2019;79(4):428-432.

24. Rohlfs AK, Friedhoff J, Bohnert A, et al. Unilateral hearing loss in children: a retrospective study and a review of the current literature. Eur J Pediatr. 2017;176(4):475-486. doi: 10.1007/s00431-016-28272.

25. Cañete O, Purdy S, Brown C, Neeff M, Thorne P. Impact of Unilateral Hearing Loss on Behavioral and Evoked Potential Measures of Auditory Function in Adults. J Am Acad Audiol. 2019;30(7):564-578.

26. Borg E, Risberg A, McAllister B, et al. Language development in hearing-impaired children. Establishment of a reference material for a 'Language test for hearing-impaired children', LATHIC. Int J Pediatr Otorhinolaryngol. 2002;65(1):15-26.

27. Kishon-Rabin L, Kuint J, Hildesheimer M, Ari-Even Roth D. Delay in auditory behaviour and preverbal vocalization in infants with unilateral hearing loss. Dev Med Child Neurol. 2015;57(12):1129-1136.

28. Bess F, Dodd-Murphy J, Parker R. Children with minimal sensorineural hearing loss: prevalence, educational performance, and functional status. Ear Hear. 1998;19(5):339-354.

29. English K, Church G. Unilateral Hearing Loss in Children: An Update for the 1990s. Lang Speech Hear Serv Sch. 1999;30(1):26-31.

30. Lieu J, Tye-Murray N, Fu Q. Longitudinal study of children with unilateral hearing loss. Laryngoscope. 2012;122(9):2088-2095.

31. Lewis DE, Valente DL, Spalding JL. Effect of Minimal/Mild Hearing Loss on Children's Speech Understanding in a Simulated Classroom. Ear Hear. 2015;36(1);136-144.

32. Kuppler K, Lewis M, Evans A. A review of unilateral hearing loss and academic performance: is it time to reassess traditional dogmata? Int J Pediatr Otorhinolaryngol. 2013;77(5):617-22.

33. Bess F, Tharpe A. Unilateral hearing impairment in children. Pediatrics. 1984;74(2):206-216.

34. Oyler R, Oyler A, Matkin N. Unilateral hearing loss: demographics and educational impact. Lang Speech Hear Serv Sch. 1988;19:201-210.

35. Lieu J, Tye-Murray N, Karzon R, Piccirillo J. Unilateral hearing loss is associated with worse speech-language scores in children. Pediatrics. 2010;125(6):1348-1355.

36. Umansky A, Jeffe D, Lieu J. The HEAR-QL: quality of life questionnaire for children with hearing loss. J Am Acad Audiol. 2011;22(10):644-53.

37. Rachakonda T, Jeffe D, Shin J, et al. Validity, discriminative ability, and reliability of the hearingrelated quality of life questionnaire for adolescents. Laryngoscope. 2014;124(2):570-578.

38. Krishnan L, Van Hyfte S. Management of unilateral hearing loss. Int J Pediatr Otorhinolaryngol. 2016;88:63-73.

39. Hartvig Jensen J, Johansen PA, Børre S. Unilateral sensorineural hearing loss in children and auditory performance with respect to right/left ear differences. Br J Audiol. 1989;23(3):207-213.

40. Vicencio S, Torrente M, Wimmer del Solar J, Délano P. Hipoacusia unilateral: bases neurobiológicas de la ambliaudia. Rev Otorrinolaringol Cir Cabeza Cuello. 2020;80(3):344-351.

41. Liu J, Zhou M, He X, Wang N. Single-sided deafness and unilateral auditory deprivation in children: current challenge of improving sound localization ability. J Int Med Res. 2020;48(1):1-13.

42. McKay S, Gravel J, Tharpe A. Amplification considerations for children with minimal or mild bilateral hearing loss and unilateral hearing loss. Trends Amplif. 2008;12(1):43-54.

43. Lieu J. Management of Children with Unilateral Hearing Loss. Otolaryngol Clin North Am. 2015;48(6):1011-1026.

44. Ministerio de Educación. JUNAEB. Norma de control de patologías de Otorrinolaringología 2017. Programa de salud del estudiante. Chile. Disponible en: https:// www.junaeb.cl/wp-content/uploads/2018/09/ Normas-de-Control-de-Patolog\%C3\%ADasOtorrinológicas-ilovepdf-compressed-1.pdf. Consultado el 18 de febrero de 2021.

45. Fitzpatrick E, Durieux-Smith A, Whittingham J. Clinical practice for children with mild bilateral and unilateral hearing loss. Ear Hear. 2010;31(3):392-400.

46. Moeller M, Hoover B, Peterson B, Stelmachowicz P. Consistency of hearing aid use in infants with earlyidentified hearing loss. Am J Audiol. 2009;18(1):1423.

47. Huttunen K, Erixon E, Löfkvist U, Mäki-Torkko E. The impact of permanent early-onset unilateral hearing impairment in children - A systematic review. Int J Pediatr Otorhinolaryngol. 2019;120:173183.

48. Lieu J. Unilateral hearing loss in children: speechlanguage and school performance. B-ENT. 2013;Suppl 21:107-115.

49. Kenworthy O, Klee T, Tharpe A. Speech recognition ability of children with unilateral sensorineural hearing loss as a function of amplification, speech stimuli and listening condition. Ear Hear. 1990;11(4):264-270.

50. Updike C. Comparison of FM auditory trainers, CROS aids, and personal amplification in unilaterally hearing impaired children. J Am Acad Audiol. 1994;5(3):204-209.

51. Appachi S, Specht J, Raol N, et al. Auditory Outcomes with Hearing Rehabilitation in Children with Unilateral Hearing Loss: A Systematic Review. Otolaryngol Head Neck Surg. 2017;157(4):565-571.

52. Dornhoffer J, Dornhoffer J. Pediatric unilateral 
sensorineural hearing loss: implications and management. Curr Opin Otolaryngol Head Neck Surg. 2016;24(6):522-528.

53. Kitterick P, Smith S, Lucas L. Hearing Instruments for Unilateral Severe-to-Profound Sensorineural Hearing Loss in Adults: A Systematic Review and Meta-Analysis. Ear Hear. 2016;37(5):495-507.

54. Di Stadio A, Dipietro L, Toffano R, et al. Working Memory Function in Children with Single Side Deafness Using a Bone-Anchored Hearing Implant: A Case-Control Study. Audiol Neurotol. 2018;23(4):238244.

55. Vlastarakos P, Nazos K, Tavoulari E, Nikolopoulos T. Cochlear implantation for single-sided deafness: the outcomes. An evidence-based approach. Eur Arch Otorhinolaryngol. 2014;271(8):2119-2126.

56. Buss E, Dillon M, Rooth M, et al. Effects of Cochlear Implantation on Binaural Hearing in Adults With Unilateral Hearing Loss. Trends Hear. 2018;22:1-15.

57. Blasco M, Redleaf M. Cochlear Implantation in unilateral sudden deafness improves tinnitus and speech comprehension: meta-analysis and systematic review. Otol Neurotol. 2014,35(8):1426-1432.

58. Dillon MT, Buss E, Anderson ML, et al. Cochlear Implantation in Cases of Unilateral Hearing Loss: Initial Localization Abilities. Ear Hear.
2017;38(5):611-619.

59. Zeitler D, Sladen D, DeJong M, Torres J, Dorman M, Carlson M. Cochlear implantation for single-sided deafness in children and adolescents. Int J Pediatr Otorhinolaryngol. 2019;118:128-133.

60. Van Wieringen A, Boudewyns A, Sangen A, Wouters J, Desloovere C. Unilateral congenital hearing loss in children: Challenges and potentials. Hear Res. 2019;372:29-41.

61. Ramos A, Borkoski-Barreiro S, Falcón J, de Miguel I, Ramos A. Single-Sided Deafness and cochlear implantation in congenital and acquired hearing loss in children. Clin Otolaryngol. 2019;44(2):138-143.

62. Sharma A, Glick H, Campbell J, Torres J, Dorman M, Zeitler D. Cortical Plasticity and Reorganization in Pediatric Single-sided Deafness Pre- and Postcochlear Implantation: A Case Study. Otol Neurotol. 2016;37(2):26-34.

63. Der C, Pardo J. Implante coclear para el manejo del tinnitus intratable en pacientes con hipoacusia unilateral. Rev Otorrinolaringol Cir Cabeza Cuello. 2010;70:265-272.

64. Hassepass F, Aschendorff A, Wesag T, et al. Unilateral deafness in children: Audiologic and subjetive assesment of hearing ability after cochlear implantation. Otol Neurotol. 2013;34: 53-60. 